\title{
A Mathematical study on the vaccination impact on the disease dynamics of $\mathrm{HBV}$
}

\author{
Jannatun Nayeem ${ }^{1}$, Chandra N. Podder ${ }^{2}$ \\ ${ }^{1}$ Department of Arts and Sciences, Ahsanullah University of Science and Technology, Dhaka, Bangladesh. \\ ${ }^{2}$ Department of Mathematics, University of Dhaka, Dhaka-1000, Bangladesh.
}

\begin{abstract}
We develop a deterministic model to understand the underlying dynamics of HBV infection at population level. The model, which incorporates the vaccination and treatment of individuals, the re-infections of latent, carrier and recovery individuals, is rigorously analyzed to gain insight into its dynamical features. The mathematical analysis reveals that the model exhibits a backward bifurcation. This phenomenon resulted due to the exogenous re-infection of $H B V$ disease. It is shown that, in the absence of such re-infection, the model has a disease-free equilibrium (DFE) which is globally asymptotically stable (GAS) using Lyapunov function and LaSalle Invariance Principle whenever the associated reproduction threshold is less than unity. Further, the model has a unique endemic equilibrium(EEP), for a special case, whenever the associate threshold quantity exceeds unity. This EEP is shown to be GAS, for a special case, using a non-linear Lyapunov function of GohVolterra type.
\end{abstract}

Keywords: Hepatitis B virus (HBV), basic reproduction number, equilibria, stability, Lyapunov function, transmission dynamics, disease endemic equilibrium.

\section{Introduction}

Hepatitis B virus (HBV) infection is a major cause of mortality and morbidity globally. Hepatitis B is a major cause of chronic liver disease and a significant public health issue. Progress in understanding of the natural history of hepatitis B made it possible the mathematical formulation of its dynamics in populations and the construction of epidemiologic models. The quest continues to identify viral factors that influences disease progression and severity as well as responses to treatment of $\mathrm{HBV}$ infection. Hepatitis B virus, abbreviated $\mathrm{HBV}$, is a species of the genus Orthohepadnavirus, which is likewise a part of the Hepadnaviridae family of viruses. This virus causes the disease hepatitis B. Approximately 5\% of the worlds population is infected with hepatitis B virus(HBV). There are approximately 400 million HBV carriers worldwide, of whom $75 \%-80 \%$ reside in Asia and the Western Pacific. Chronic hepatitis $\mathrm{B}(\mathrm{CHB})$ is responsible for 1 million deaths per year globally (Tibbs and Simth (2001)). Hepatitis is common disease world wide, specially, in Bangladesh. As a country of the Asia Pacific region Bangladesh is considered to be a high risk country for developing hepatitis A and B(Parvin et al (2011)). In particular, HBV lead to chronic disease in hundreds of millions of people and the most common cause of liver cirrhosis and cancer (WHO, 2011). As a south East Asian country Bangladesh is considered endemic for hepatitis B virus (HBV) infection (Chowdhury et al (2009)). Bangladesh is a densely populated country where about 10 million people are chronically infected with hepatitis B virus (HBV)(Ahmad et al (2008)). Bangladesh is a densely populated country with about 150 million people, where HBsAg positivity in the healthy adult population is 7.2\%-7.5\% (Islam et al (1984), Khan and Ahmad (1996)). In Bangladesh, most HBV infections occur in childhood as suggested by the high rate of interfamilial HBV infection, history of low rate of acute hepatitis and large number of younger populations affected.

Hepatitis has become an issue of global importance, and in Bangladesh, hepatitis B virus infections still remain a public health problem. Those who have the infection may develop chronic hepatitis eventually leading to cirrhosis and hepatocellular carcinoma. In a study conducted by the World Health Organization, an estimated 2 billion people have been infected with hepatitis B at some time in their life worldwide(WHO(1998)).The prevalence of hepatitis B infection varies in different regions. In Bangladesh, the prevalence of $\mathrm{HBs} A g(+)$ reported a value of $8 \%$ in IV drug users and a value of $9.7 \%$ in commercial sex workers and $5.9 \%$ in truck drivers(Gibney et al (2001)). Chronic infection developed in $90 \%$ of neonates infected with hepatitis B; in 20\% $50 \%$ of children below the age of 10 but in only $2 \%$ to $7 \%$ of adults (Hsu Hy et al (1992)). The infection with Hepatitis B virus (HBV) is a major health problem in the world. The WHO has reported that more than 2 billion people worldwide has been infected by HBV. There are over 350 millions who are chronically infected with HBV. Mathematical analysis of the HBV dynamics not only provide important quantitative insights into the pathogenesis, but also lead to design treatment strategies which would more effectively bring the infection under control. Hepatitis B dynamics from the point of view of quantitative epidemiology is far from being simple and straightforward matter due to the chronicity of infection. Using modeling techniques and the new knowledge on biology and epidemiology, the efforts have been made to construct such models and to use then in solving 
practical public health problems related to the effective control of Hepatitis B. HBV is transmitted by percutaneous and mucous membrane exposures to infectious blood and body fluids that contain blood. Viral hepatitis is the commonest liver disease in Bangladesh. About 10 million people in Bangladesh have been suffering from hepatitis B. A proportion of them are hepatitis B carrier and another proportion is affected by the long standing consequences of this infection. The treatment of hepatitis B possesses a great challenge (Ahad and Alim (2006)). HBV is an important cause of liver disease in Bangladesh and is responsible for $19.0 \%$ to $35.0 \%$ of acute viral hepatitis, $35.7 \%$ of acute liver failure, $33.3 \%$ to $40.5 \%$ of chronic hepatitis and $46.8 \%$ of hepatocellular carcinoma. Chronic HBV infection is most commonly defined as being present when a person tests positive for HBsAg for an at least six months (Lok et al (2001)). Hepatitis B virus is encountered sporadically year- round in Bangladesh. It results in a wide range of varying liver diseases with asymptomatic acute hepatitis at one end and hepatocellular carcinoma (HCC) at the other end of the spectrum.

Using mathematical models to enhance our understanding of the dynamics of chronic viral infections has been proved fruitful. Using mathematical models to interpret experimental and clinical results has made a significant contribution to the fields of anti-HIV, HBV and HCV infections (Perelson et al (1996),LeenhEEr and Smith (2003)).The aim of this study was to evaluate the different characteristics of CHB patients and to examine the relationship between them. So, early detection of HBsAg among the healthy population can save many lives from dangerous complication of cirrhosis and hepatocellular carcinoma by proper treatment. The goal of this study was to detect HBsAg positive donors and advised them for treatment accordingly.

Progress in understanding of the natural history of hepatitis B made it possible the mathematical formulation of its dynamics in population and the construction of epidemiologic models. The paper is organized as follows. Model formulation is presented in section 2. In section 3, we analyze the qualitative property of the model, we evaluate the basic reproduction number and determine the existence and stability of both the disease free and disease endemic equilibrium of the model. In section 4, we discuss the global stability of DFE of the model. In section 5 we illustrate the existence of unique endemic equilibrium points for special case. A brief discussion on local stability of endemic equilibrium points (EEP)is given in section 6 . Then, in section 7, we perform the global stability of EEP. And lastly we conclude our discussion and numerical result simulation in last sections.

\section{Formulation of Model}

We design a mathematical model to understand the transmission dynamics and prevalence of HBV. The model is constructed based on the characteristics of HBV transmission and the model of Medley(2001) . Note that, there is considered only five epidemiological groups and those did not distinguish the recovery and vaccinated subgroups (Zou et al (2010)). In our model, the latent, carrier and recovered individuals may be reinfected at any time by the contact of HBV infected individuals. Some studies (Mandell et al (1979), Shepard et al (2006)) indicated that acute hepatitis B could be found today in newborns of infected mothers. The total homogeneously-mixing population at time $\mathrm{t}$, is denoted by $N(t)$. The total host population is sub-divided into six epidemiological groups: the susceptible individuals $(X(t))$, individuals who are protective immunized by vaccination $(Y(t))$, infected but not yet infectious (latent) $(L(t))$, acute infected individuals $(I(t))$, chronic HBV carriers $(C(t))$ and acquiring treatment recovered individuals $(R(t))$ but can get infection at any time by contact of HBV infected individuals, so that

$$
N(t)=X(t)+Y(t)+L(t)+I(t)+C(t)+R(t) .
$$

The susceptible population is decreased by infection, which can be acquired following effective contact with infectious individuals in acute (I) and chronic carriers(C), at a rate $\lambda$ given by

$$
\lambda=\beta(I+\eta C) / N .
$$

In (1); $\beta$ represents the effective contact rate (i.e. contact capable of leading to HBV infection); $\eta$ is the modification parameter; the infectiousness of carriers relative to acute infections. We assume that $0<\eta<1$.

The susceptible individuals consists of un protective immunized population with portion of infected carrier mothers $\mu \omega(1-v C)$, that means newborns are un immunized and become susceptible individuals and decreased by vaccination ( at a rate $\sigma$ ) and natural death ( at a rate $\mu_{1}$ ) and increased by unsuccessful vaccination ( at a rate $(1-\sigma)$ ). Thus the rate of change of the susceptible population is given by

$$
\frac{d X}{d t}=\mu \omega(1-v C)-\lambda X-\sigma X+(1-\sigma) Y-\mu_{1} X
$$


The protective immunized individual is increased by the recruitment of newborns successfully immunized at rate $\mu(1-\omega)$ and vaccinated susceptible individuals and decreased by unsuccessful vaccination ( at a rate $(1-\sigma))$ and natural death ( at a rate $\left.\mu_{1}\right)$. Thus the rate of change of protective immunized population is given by

$$
\frac{d Y}{d t}=\mu(1-\omega)+\sigma X-(1-\sigma) Y-\mu_{1} Y .
$$

$v$ is the portion of unimmunized children born to carrier mothers that have been infected, which means that babies have infected in perinatal infection and access to latent class. A fraction $\xi$, of the newly infected individuals are assumed to show no disease symptoms initially. These individuals (known as slow progressors) are moved to latent class (L). The remaining fraction, $1-\xi$, of the newly infected individuals are assumed to immediately display disease symptoms(fast progressors) and are moved to the acute class (I). The population of latent individuals is decreased by the progression of latent individuals to acute HBV ( at a rate $\varepsilon_{1}$ ) and at $\omega_{1}$ fraction of latent individuals who develop symptoms and are detected. These individuals is also reduced by the transmission probability, $\beta_{1}$ with transfer rate $\varepsilon_{2}$ from latent to carrier class and re-infection ( at a rate $\omega_{2} \psi_{\mathrm{e}} \lambda$, where $\psi_{\mathrm{e}}<1$ accounts for the assumption that latent individuals have reduced infection rate in comparison to susceptible individuals and $\omega_{2}$ is the fraction of re-infected latent individuals who are detected. It is further reduced by natural death (at a rate $\left.\mu_{1}\right)$. Thus

$$
\frac{d L}{d t}=\mu \omega \nu C+\xi \lambda X-\omega_{1} \varepsilon_{1} L-\beta_{1} \varepsilon_{2} L-\omega_{2} \psi_{e} \lambda L-\mu_{1} L
$$

The population of acute HBV individuals is increased by the infection of fast progressors(at the rate (1 $-\xi) \lambda$ and the development of symptoms by latent individuals (at the rate $\omega_{1} \varepsilon_{1}$ ). It is further increased by the reinfection of latent individuals ( at the rate $\omega_{2} \psi_{\mathrm{e}} \lambda$ ) and re-infection of carrier individuals ( at the rate $\omega_{3} \psi_{\mathrm{c}} \lambda$ ), where $\omega_{3}$ is the fraction of re-infected carriers individuals who are detected. The acute HBV individuals is decreased by the transmission probability, $\beta_{2}$ with the transfer rate $\varepsilon_{3}$ from acute to carrier class and acquiring treatment ( at the rate $t_{1}$ ), the individuals are transmitted with the probability, $\beta_{3}$ from acute to recover class. It is also reduced by disease induced death ( at the rate $\mu_{2}$ ). Thus the governing equation is

$$
\frac{d I}{d t}=(1-\xi) \lambda X+\omega_{1} \varepsilon_{1} L+\omega_{2} \psi_{e} \lambda L+\omega_{3} \psi_{c} \lambda C-\beta_{2} \varepsilon_{3} I-\beta_{3} t_{1} I-\mu_{2} I
$$

The population of chronic carrier individuals increases by the transfer rate, $\beta_{1} \varepsilon_{2}$ from latent to carrier and the transfer rate, $\beta_{2} \varepsilon_{3}$ from acute to carrier and re-infection of recovered individuals ( at the rate $\omega_{4} \psi_{\mathrm{r}} \lambda$ ) where $\omega_{4}$ is the fraction of re-infected recovered individuals who are detected. The population is decreased by re-infection of carrier individuals ( at the rate $\omega_{3} \psi_{\mathrm{c}} \lambda$ ) from carrier to acute HBV infected individuals and acquiring treatment ( at the rate $t_{1}$ ), with the transmission probability, $\beta_{4}$ from carrier to recovered individuals. It is also reduced by natural death ( at a rate $\mu_{1}$ ) and disease induced death ( at the rate $\mu_{2}$ ). Hence

$$
\frac{d C}{d t}=\beta_{1} \varepsilon_{2} L+\beta_{2} \varepsilon_{3} I-\omega_{3} \psi_{c} \lambda C-\beta_{4} t_{1} C-\mu_{1} C-\mu_{2} C+\omega_{4} \psi_{r} \lambda R
$$

Recovery means recovery from illness acquiring treatment ( at the rate $t_{1}$ ) with transmission probability, $\beta_{3}$ and $\beta_{4}$ from acute to recovered and from carrier to recovered. But recovery rate is temporary and after some times these individuals can get re infection and go back to the carrier class ( at the rate $\omega_{4} \psi_{\mathrm{r}} \lambda$ ) and decreased by natural death ( at a rate $\mu_{1}$ ). Hence

$$
\frac{d R}{d t}=\beta_{3} t_{1} I+\beta_{4} t_{l} C-\omega_{4} \psi_{r} \lambda R-\mu_{l} R
$$

Based on the characteristics of HBV transmission the non-linear differential equations ( associated variables and parameters are described in Table 1) are given by

$$
\begin{aligned}
& \frac{d X}{d t}=\mu \omega(1-v \mathrm{C})-\lambda \mathrm{X}-\sigma \mathrm{X}+(1-\sigma) \mathrm{Y}-\mu_{1} \mathrm{X}, \\
& \frac{d Y}{d t}=\mu(1-\omega)+\sigma \mathrm{X}-(1-\sigma) \mathrm{Y}-\mu_{1} \mathrm{Y}, \\
& \frac{d L}{d t}=\mu \omega \nu \mathrm{C}+\xi \lambda \mathrm{X}-\omega_{1} \varepsilon_{1} \mathrm{~L}-\beta_{1} \varepsilon_{2} \mathrm{~L}-\omega_{2} \psi_{\mathrm{e}} \lambda \mathrm{L}-\mu_{1} \mathrm{~L}, \\
& \frac{d I}{d t}=(1-\xi) \lambda \mathrm{X}+\omega_{1} \varepsilon_{1} \mathrm{~L}+\omega_{2} \psi_{\mathrm{e}} \lambda \mathrm{L}+\omega_{3} \psi_{\mathrm{c}} \lambda \mathrm{C}-\beta_{2} \varepsilon_{3} \mathrm{I}-\beta_{3} \mathrm{t}_{1} \mathrm{I}-\mu_{2} \mathrm{I},
\end{aligned}
$$




$$
\begin{aligned}
& \frac{d C}{d t}=\beta_{1} \varepsilon_{2} \mathrm{~L}+\beta_{2} \varepsilon_{3} \mathrm{I}-\omega_{3} \psi_{\mathrm{c}} \lambda \mathrm{C}-\beta_{4} \mathrm{t}_{1} \mathrm{C}-\mu_{1} \mathrm{C}-\mu_{2} \mathrm{C}+\omega_{4} \psi_{\mathrm{r}} \lambda \mathrm{R}, \\
& \frac{d R}{d t}=\beta_{3} \mathrm{t}_{1} \mathrm{I}+\beta_{4} \mathrm{t}_{1} \mathrm{C}-\omega_{4} \psi_{\mathrm{r}} \lambda \mathrm{R}-\mu_{1} \mathrm{R} .
\end{aligned}
$$

The important features of the model (8) are that it:

(i) allows for disease transmission by individuals in the latent (L), acute (I) and carrier (C) classes;

(ii) latent individuals show their disease symptoms (at the rate $\omega_{1}$ ) and move to the acute classes;

(iii) allows for the re-infection of latent, carrier and recovered individuals (at the rates $\psi_{\mathrm{e}} \lambda, \psi_{\mathrm{c}} \lambda$ and $\psi_{\mathrm{r}} \lambda$ respectively);

(iv) allows for slow progression (at the rate $\xi \lambda$ )to the latent class and fast progression (at the rate $(1-\xi) \lambda$ ) to acute disease;

(v) of re-infection individuals who develop symptoms (due to re-infection of disease) into the acute and carrier classes ;

(vi) allows for the treatment (at a rate $\mathrm{t}_{1}$ ), temporarily release from the disease into acute and carrier classes and moves to recovered class and can get re-infection at any time.

The model (8) extends the models in many of the aforementioned studies (Pang et al (2010), Zhaoa et al (2010)) by including treatment to the infected individuals. Furthermore it extends the study (Pang et al (2010)) using standard incidence and re-infection. Also, the model offers additional extensions to many of the earlier models by incorporating the slow and fast progression aspect of HBV disease (by splitting the number of new infected individuals into the latent (L) and acute(C) classes). The study further contributes to the literature by carrying out a detailed rigorous analysis of the model( $(8)$. Here we consider the protective immunized new born carrier from chronic carrier to latent class (Pang et al (2010)). Because some studies (Mandell et al (1979), Shepard et al (2006)) indicated that acute Hepatitis B could be found today in newborns of infected mothers. According to the change of the model (8), we analyze the dynamical behavior of our suggested model.

Variables
$\mu$
$\omega$
$(1-\omega)$
$\sigma$
$(1-\sigma)$
$\mu_{1}$
$\mu_{2}$
$\lambda$
$\beta$
$\eta$
$\varepsilon_{1}$
$\varepsilon_{2}$
$\varepsilon_{3}$
$\beta_{1}$
$\beta_{2}$
$\beta_{3}$
$\beta_{4}$
$\mathrm{t}_{1}$
$(1-\xi)$

$\psi_{\mathrm{e}} \lambda$
$\omega_{1}$
$\omega_{2}$
$\omega_{3}$
$\omega_{4}$

Table 1: Description of variables of the model:

\section{Description}

birth rate

proportion of birth rate without protective immunity proportion of birth rate with protective immunity vaccination rate

unsuccessful vaccination rate natural mortality rate mortality rate due to $\mathrm{HBV}$ force of infection effective contact rate for HBV infection rate of infectiousness of carrier relative to acute infections transfer rate from latent to acute transfer rate from latent to carrier transfer rate from acute to carrier transmission probability from latent to carrier transmission probability from acute to carrier transmission probability from acute to recovered acquiring treatment transmission probability from carrier to recovered acquiring treatment rate of treatment

A fraction of the newly infected individuals who are fast progressors assumed to immediately display disease symptoms re infection rate, latent individuals have reduced infection rate in comparison to wholly susceptible individuals

fraction of latent individuals who develop symptoms are infected fraction of re infected latent individuals who are infected fraction of re infected carrier individuals who are infected fraction of re infected recovered individuals who are infected 
Table 2: Data summary of parameters of the model:

$\begin{array}{cc}\text { Parameters } & \text { Values } \\ \mu & 0.0247 \\ \omega & 0.5 \text { (Assumed) } \\ (1-\omega) & 0.5 \text { (Assumed) } \\ \sigma & 0-1 \\ (1-\sigma) & \text { Assumed } \\ \mu_{1} & 0.00693 \\ \mu_{2} & 0.31 \text { (Assumed } 0.2) \\ \beta & 0.98 \text { (Assumed) } \\ \eta & 0-1 \\ \varepsilon_{1} & 6 / 365 \\ \varepsilon_{2} & 8 / 365 \\ \varepsilon_{3} & 4 / 365 \\ \beta_{1} & 0.1 \\ \beta_{2} & 0.1 \\ \beta_{3} & 0.5 \text { (Assumed) } \\ \beta_{4} & 0.95 \text { (Assumed) } \\ \mathrm{t}_{1} & 0.0525 \\ \xi & 0.67 \text { (Assumed } 0.7) \\ \psi_{\mathrm{e}}, \psi_{\mathrm{c}}, \psi_{\mathrm{r}} & 0.297 \\ \omega_{1} & 0.045 \text { (Assumed) } \\ \omega_{2} & 0.4 \text { (Assumed) } \\ \omega_{3} & 0.6 \text { (Assumed) } \\ \omega_{4} & 0.6 \text { (Assumed) }\end{array}$

\section{Analysis of the Model}

Disease Free Equilibrium(DFE): The model (8) has a DFE, obtained by setting the right-hand sides of the equations of the model to zero, given by

$\varepsilon_{0}=(\mathrm{X}, \mathrm{Y}, \mathrm{L}, \mathrm{I}, \mathrm{C}, \mathrm{R})=\left(\frac{\mu(1-\sigma)+\mu \mu_{1} \omega}{\mu_{1}+\mu_{1}^{2}}, \frac{\mu \sigma+\mu \mu_{1}(1-\omega)}{\mu_{1}+\mu_{1}^{2}}, 0,0,0,0\right)$

The stability of the DFE, $\varepsilon_{0}$, will be analyzed using the next generation method (Driessche and Watmough (2002)). The non-negative matrix F (of the new infection terms) and the non-singular M-matrix V (of the remaining transfer terms) are given, respectively, by:

$$
\begin{gathered}
\mathrm{F}=\left[\begin{array}{ccc}
0 & \frac{\xi \beta x_{4}}{N} & \frac{\xi \beta \eta x_{4}}{N} \\
0 & \frac{(1-\xi) \beta x_{4}}{N} & \frac{(1-\xi) \beta \eta x_{4}}{N} \\
0 & 0 & 0
\end{array}\right], \\
\mathrm{V}=\left[\begin{array}{ccc}
k_{1} & 0 & -\mu \omega v \\
-\omega_{1} \varepsilon_{1} & k_{2} & 0 \\
-\beta_{1} \varepsilon_{2} & -\beta_{2} \varepsilon_{3} & k_{3}
\end{array}\right],
\end{gathered}
$$

where, $\mathrm{k}_{1}=\omega_{1} \varepsilon_{1}+\beta_{1} \varepsilon_{2}+\mu_{1}, \mathrm{k}_{2}=\beta_{2} \varepsilon_{3}+\beta_{3} \mathrm{t}_{1}+\mu_{2}$ and $\mathrm{k}_{3}=\beta_{4} \mathrm{t}_{1}+\mu_{1}+\mu_{2}$ and $\mathrm{x}_{4}=\frac{\mu(1-\sigma)+\mu \mu_{1} \omega}{\mu_{1}+\mu_{1}^{2}}$.

The associated reproduction number, denoted by $\mathrm{R}_{0}$, is given by $\mathrm{R}_{0}=\rho\left(\mathrm{FV}^{-1}\right)$, where $\rho$ denotes the spectral radius (dominant eigenvalue in magnitude)- of the next generation matrix $\mathrm{FV}^{-1}$. It follows that 


$$
\mathrm{R}_{0}=\frac{\beta x_{4}\left[k_{1} k_{3}(1-\xi)-\beta_{1} \mu \omega v(1-\xi)+\eta k_{1} \beta_{2}+\xi \eta k_{4}+\xi \omega_{1} k_{3}\right]}{N\left(k_{1} k_{2} k_{3}-\mu \omega v k_{4}\right)},
$$

It can also be rewritten as (after some algebraic manipulation),

with,

$$
\mathrm{R}_{0}=\frac{\beta x_{4} k_{1} k_{3}(1-\xi)+\beta x_{4} \eta \beta_{2}(1-\xi) k_{1}+\beta x_{4} \xi \eta k_{4}+\beta x_{4} \xi \omega_{1} k_{3}+N \mu \omega v k_{4}}{N k_{1} k_{2} k_{3}+\beta_{1}(1-\xi) \beta x_{4} \mu \omega v},
$$

$$
\begin{aligned}
& \mathrm{k}_{1}=\omega_{1} \varepsilon_{1}+\beta_{1} \varepsilon_{2}+\mu_{1}, \\
& \mathrm{k}_{2}=\beta_{2} \varepsilon_{3}+\beta_{3} \mathrm{t}_{1}+\mu_{2}, \\
& \mathrm{k}_{3}=\beta_{4} \mathrm{t}_{1}+\mu_{1}+\mu_{2}, \\
& \mathrm{k}_{4}=\beta_{1} \mathrm{k}_{2}+\omega_{1} \beta_{2} .
\end{aligned}
$$

Hence, the result below follows from Theorem 2 of (Driessche and Watmough (2002)) .

Lemma 1. The Disease free equilibrium, $\varepsilon_{0}$ of the model (8), is locally asymptotically stable (LAS) if $\mathrm{R}_{0}<1$, and unstable if $\mathrm{R}_{0}>1$.

The threshold quantity, $\mathrm{R}_{0}$, is the reproduction number for the model. The epidemiological implication of Lemma 1 is that HBV spread can be effectively controlled in the community (when $\mathrm{R}_{0}<1$ ) if the initial sizes of the populations of the model are in the basin of attraction of the disease free equilibrium $\varepsilon_{0}$.

Since we have considered HBV model with re-infection in some stages, are often shown the backward bifurcation, where the stable DFE co-exists with a stable endemic equilibrium when the associated reproduction threshold $\left(\mathrm{R}_{0}\right)$ is less than unity, it is instructive to determine whether or not the model (8) also exhibits this dynamical feature. This is investigated below.

Theorem 1. The model (8) undergoes a backward bifurcation at $R_{0}=1$ if the inequality (14) holds.

Proof . The proof of Theorem 1, which is based on the use of centre manifold theory (Driessche and Watmough (2002), Carr (1981), Dushoff et al (1998)). The backward bifurcation phenomenon of the model (8) is numerically illustrated below. it is convenient to let $\mathrm{X}=\mathrm{x}_{1}, \mathrm{Y}=\mathrm{x}_{2}, \mathrm{~L}=\mathrm{x}_{3}, \mathrm{I}=\mathrm{x}_{4}, \mathrm{C}=\mathrm{x}_{5}$ and $\mathrm{R}=\mathrm{x}_{6}$, so that $\mathrm{N}=$ $\mathrm{x}_{1}+\mathrm{x}_{2}+\mathrm{x}_{3}+\mathrm{x}_{4}+\mathrm{x}_{5}+\mathrm{x}_{6}$. Further, by introducing the vector notation $\mathbf{x}=\left(\mathrm{x}_{1}, \mathrm{x}_{2}, \mathrm{x}_{3}, \mathrm{x}_{4}, \mathrm{x}_{5}, \mathrm{x}_{6}\right)^{\mathrm{T}}$, the model (8) can be written in the form

$$
\begin{gathered}
\frac{d x}{d t}=\mathrm{F}(\mathbf{x}), \text { where } \mathrm{F}=\left(\mathrm{f}_{1}, \mathrm{f}_{2}, \mathrm{f}_{3}, \mathrm{f}_{4}, \mathrm{f}_{5}, \mathrm{f}_{6}\right)^{\mathrm{T}}, \text { as follows: } \\
\frac{d x_{1}}{d t}=\mathrm{f}_{1}=\mu \omega\left(1-v \mathrm{x}_{5}\right)-\frac{\beta\left(x_{4}+\eta x_{5}\right) x_{1}}{x_{1}+x_{2}+x_{3}+x_{4}+x_{5}+x_{6}}-\sigma \mathrm{x}_{1}+(1-\sigma) \mathrm{x}_{2}-\mu_{1} \mathrm{x}_{1}, \\
\frac{d x_{2}}{d t}=\mathrm{f}_{2}=\mu(1-\omega)+\sigma \mathrm{x}_{1}-(1-\sigma) \mathrm{x}_{2}-\mu_{1} \mathrm{x}_{2}, \\
\frac{d x_{3}}{d t}=\mathrm{f}_{3}=\mu \omega \mathrm{x}_{5}+\frac{\xi \beta\left(x_{4}+\eta x_{5}\right) x_{1}}{x_{1}+x_{2}+x_{3}+x_{4}+x_{5}+x_{6}}-\omega_{1} \varepsilon_{1} \mathrm{x}_{3}-\beta_{2} \varepsilon_{2} \mathrm{x}_{3} \\
\frac{d x_{4}}{d t}=\mathrm{f}_{4}=\frac{(1-\xi) \beta\left(x_{4}+\eta x_{5}\right) x_{1}}{x_{1}+x_{2}+x_{3}+x_{4}+x_{5}+x_{6}}+\omega_{1} \varepsilon_{1} \mathrm{x}_{3}-\beta_{2} \varepsilon_{3} \mathrm{x}_{4}-\beta_{3} \mathrm{t}_{1} \mathrm{x}_{4}+ \\
\frac{\omega_{2} \psi\left(x_{e} \beta\left(x_{4}+\eta x_{5}\right) x_{3}\right.}{x_{1}+x_{2}+x_{3}+x_{4}+x_{5}+x_{6}}+\frac{\omega_{3} \psi_{c} \beta\left(x_{4}+\eta x_{5}\right) x_{5}}{x_{1}+x_{2}+x_{3}+x_{4}+x_{5}+x_{6}}-\mu_{2} \mathrm{x}_{4}, \\
\frac{d x_{5}}{d t}=\mathrm{f}_{5}= \\
\beta_{1} \varepsilon_{2} \mathrm{x}_{3}+\beta_{2} \varepsilon_{3} \mathrm{x}_{4}-\frac{\omega_{3} \psi_{c} \beta\left(x_{4}+\eta x_{5}\right) x_{5}}{x_{1}+x_{2}+x_{3}+x_{4}+x_{5}+x_{6}}-\beta_{4} \mathrm{t}_{1} \mathrm{x}_{5}+
\end{gathered}
$$




$$
\begin{gathered}
\frac{\omega_{4} \psi_{r} \beta\left(x_{4}+\eta x_{5}\right) x_{6}}{x_{1}+x_{2}+x_{3}+x_{4}+x_{5}+x_{6}}-\left(\mu_{1}+\mu_{2}\right) \mathrm{x}_{5} \\
\frac{d x_{6}}{d t}=\mathrm{f}_{6}=\beta_{3} \mathrm{t}_{1} \mathrm{x}_{4}+\beta_{4} \mathrm{t}_{1} \mathrm{x}_{5}-\frac{\omega_{4} \psi_{r} \beta\left(x_{4}+\eta x_{5}\right) x_{6}}{x_{1}+x_{2}+x_{3}+x_{4}+x_{5}+x_{6}}-\mu_{1} \mathrm{x}_{6} .
\end{gathered}
$$

where, $\lambda=\frac{\beta\left(x_{4}+\eta x_{5}\right)}{N}$. The Jacobian of the system (10), or equivalently (8), at the DFE $\left(\varepsilon_{0}\right)$ is given by

$$
J\left(\varepsilon_{0}\right)=\left[\begin{array}{cccccc}
-\left(\sigma+\mu_{1}\right) & 1-\sigma & 0 & -A & -(\mu \omega v+B) & 0 \\
\sigma & -\left(1-\sigma+\mu_{1}\right) & 0 & 0 & 0 & 0 \\
0 & 0 & -k_{1} & \xi A & \mu \omega v+\beta B & 0 \\
0 & 0 & \omega_{1} \varepsilon_{1} & (1-\xi) A-k_{2} & (1-\xi) B & 0 \\
0 & 0 & \beta_{1} \varepsilon_{2} & \beta_{2} \varepsilon_{3} & -k_{3} & 0 \\
0 & 0 & 0 & \beta_{3} t_{1} & \beta_{4} t_{1} & -\mu_{1}
\end{array}\right],
$$

where $\mathrm{A}=\frac{\beta x_{1}{ }^{*}}{x_{1}{ }^{*}+x_{2}{ }^{*}} ; \mathrm{B}=\frac{\beta \eta x_{1}{ }^{*}}{x_{1}{ }^{*}+x_{2}{ }^{*}} ; \mathrm{k}_{1}=\omega_{1} \varepsilon_{1}+\beta_{1} \varepsilon_{2}+\mu_{1} ; \mathrm{k}_{2}=\beta_{2} \varepsilon_{3}+\beta_{3} \mathrm{t}_{1}+\mu_{2}$ and $\mathrm{k}_{3}=\beta_{4} \mathrm{t}_{1}+\mu_{1}+\mu_{2}$.

from which it can be shown (as before) that:

$$
\mathrm{R}_{0}=\frac{\beta x_{4} k_{1} k_{3}(1-\xi)+\beta x_{4} \eta \beta_{2}(1-\xi) k_{1}+\beta x_{4} \xi \eta k_{4}+\beta x_{4} \xi \omega_{1} k_{3}+N \mu \omega v k_{4}}{N k_{1} k_{2} k_{3}+\beta_{1}(1-\xi) \beta x_{4} \mu \omega v}
$$

which is as defined in Section 3. Consider the case when $R_{0}=1$. Suppose, further that $\beta$ is chosen as a bifurcation parameter. Solving (11) for $\mathrm{R}_{0}=1$ gives

$$
\beta=\beta^{*}=\frac{N^{*} k_{1} k_{2} k_{3}+\beta_{1} x_{4}{ }^{*} \mu \omega \nu(1-\xi)}{x_{4}{ }^{*} k_{1} k_{3}(1-\xi)+x_{4}{ }^{*} \eta \beta_{2}(1-\xi) k_{1}+x_{4}{ }^{*} \xi \eta k_{4}+x_{4}{ }^{*} \xi \omega_{1} k_{3}+N^{*} \mu \omega \nu k_{4}}
$$

where $\mathrm{k}_{1}, \mathrm{k}_{2}, \mathrm{k}_{3}, \mathrm{k}_{4}, x_{4}{ }^{*}$ and $N^{*}$ are defined in section 3 . The above linearized system, of the transformed system (10) with $\beta=\beta^{*}$, has a zero eigenvalue which is simple. Hence the Centre Manifold Theory (Carr (1981)) can be used to analyze the dynamics of (10) near $\beta=\beta^{*}$.

Eigenvectors of $\left.J\left(\varepsilon_{0}\right)\right|_{\beta=\beta^{*}}$

To analyze the dynamics of (10); we compute the eigenvalues of the jacobian of (10) at the disease free equilibrium (DFE). It can be shown that this jacobian has a left eigenvector given by: $\mathrm{V}=\left(\mathrm{v}_{1}, \mathrm{v}_{2}, \mathrm{v}_{3}, \mathrm{v}_{4}, \mathrm{v}_{5}, \mathrm{v}_{6}\right)^{\mathrm{T}}$ where,

$$
\begin{gathered}
\mathrm{v}_{1}=0, \\
\mathrm{v}_{2}=0, \\
\mathrm{v}_{3}=\frac{\omega_{1} \varepsilon_{1} v_{4}+\beta_{1} \varepsilon_{2} v_{5}}{k_{1}}, \\
\mathrm{v}_{4}=\text { free, } \\
\mathrm{v}_{5}=\text { free, } \\
\mathrm{v}_{6}=0
\end{gathered}
$$

and a right eigenvector given by : $\mathrm{W}=\left(\mathrm{w}_{1}, \mathrm{w}_{2}, \mathrm{w}_{3}, \mathrm{w}_{4}, \mathrm{w}_{5}, \mathrm{w}_{6}\right)^{\mathrm{T}}$ where,

$$
\begin{gathered}
\mathrm{w}_{1}=\frac{\left(1-\sigma+\mu_{1}\right) w_{2}}{\sigma}, \\
\mathrm{w}_{2}=\text { free, }
\end{gathered}
$$




$$
\begin{gathered}
\mathrm{w}_{3}=\frac{\xi A w_{4}+(\mu \omega \nu+\xi B) w_{5}}{k_{1}}, \\
\mathrm{w}_{4}=\text { free, } \\
\mathrm{w}_{5}=\text { free, } \\
\mathrm{w}_{6}=\frac{\beta_{3} t_{1} w_{4}+\beta_{4} t_{1} w_{5}}{\mu_{1}} .
\end{gathered}
$$

The theorem 4.1 in (Chavez and Song (2004)),reproduced below for convenience, will be used to prove the presence of backward bifurcation of (10).

Theorem 2. (Castillo-Chavez and Song(2004)). Consider the following general system of ordinary differential equations with a parameter $\phi$

$$
\frac{d x}{d t}=f(x, \phi), f: R^{n} \times R \rightarrow R^{n} \text { and } f \in C^{2}\left(R^{n} \times R\right),
$$

where 0 is an equilibrium point of the system (that is, $f(0, \phi) \equiv 0$ for all $\phi$ )

1. A $=D_{x} f(0,0)=\left(\frac{\partial f_{i}}{\partial x_{j}}(0,0)\right)$ is the linearization matrix of the system around the equilibrium 0 with $\phi$ evaluated at 0 ;

2. Zero is a simple eigenvalue of A and all other eigenvalues of A have negative real parts;

3. Matrix A has a right eigenvector $w$ and a left eigenvector $v$ corresponding to the zero

eigenvalue.

Let $f_{k}$ be the $k$ - th component of $f$ and

$$
\begin{aligned}
& a=\sum_{k, i, j=1}^{n} v_{k} w_{i} w_{j} \frac{\partial^{2} f_{k}}{\partial x_{i} \partial x_{j}}(0,0), \\
& b=\sum_{k, i=1}^{n} v_{k} w_{i} \frac{\partial^{2} f_{k}}{\partial x_{i} \partial \phi}(0,0) .
\end{aligned}
$$

Then the local dynamics of the system around the equilibrium point 0 is totally determined by the signs of $a$ and $b$. Particularly, if $a>0$ and $b>0$, then a backward bifurcation occurs at $\phi=0$.

\section{Computations of $a$ and $b$}

For the partial derivatives in the equation for a in (12) gives :

$$
\begin{gathered}
a=\sum_{k, i, j=1}^{n} v_{k} w_{i} w_{j} \frac{\partial^{2} f_{k}}{\partial x_{i} \partial x_{j}} \\
=\frac{-2 \beta^{*}\left(w_{5} \eta+w_{4}\right)}{\left(x_{1}^{*}+x_{2}\right)^{2}}\left[(1-\xi)\left(v_{4} w_{5} x_{1}^{*}+v_{4} w_{2} x_{1}^{*}+v_{4} w_{4} x_{1}{ }^{*}+v_{4} w_{3} x_{1}{ }^{*}+v_{4} w_{6} x_{1}{ }^{*}+v_{4} w_{1} x_{2}{ }^{*}\right)\right. \\
+\left(x_{1}^{*}+x_{2}{ }^{*}\right)\left(v_{4} w_{5} \omega_{3} \psi_{c}-v_{5} w_{5} \omega_{3} \psi_{c}+v_{4} w_{3} \omega_{2} \psi_{e}+v_{5} w_{6} \omega_{4} \psi_{r}-v_{3} w_{3} \omega_{2} \psi_{e}\right)-v_{3} w_{5} \xi x_{1}{ }^{*} \\
\left.-v_{3} w_{2} \xi x_{1}^{*}-v_{3} w_{4} \xi x_{1}{ }^{*}-v_{3} w_{1} \xi x_{2}{ }^{*}+v_{3} w_{6} \xi x_{1}^{*}+v_{3} w_{3} \xi x_{1}^{*}\right]
\end{gathered}
$$

from which it can be shown that $a>0$ if

$$
\mathrm{B} 1>\mathrm{B} 2
$$

where $B_{1}=v_{3} \xi x_{1}^{*}\left(w_{3}+w_{6}\right)+\left(x_{1}^{*}+x_{2}^{*}\right)\left(v_{4} w_{5} \omega_{3} \psi_{c}+v_{4} w_{3} \omega_{2} \psi_{e}+v_{5} w_{6} \omega_{4} \psi_{r}\right)$,

$$
\begin{aligned}
B_{2}= & v_{4} x_{1}{ }^{*}\left[(1-\xi)\left(w_{1}+w_{2}+w_{3}+w_{4}+w_{5}+w_{6}\right)\right]+v_{3} \xi x_{1}{ }^{*}\left(w_{2}+w_{4}+w_{5}\right)+v_{3} w_{1} \xi x_{2}{ }^{*} \\
& +\left(x_{1}{ }^{*}+x_{2}{ }^{*}\right)\left(v_{5} w_{5} \omega_{3} \psi_{c}+v_{3} w_{3} \omega_{2} \psi_{e}\right) .
\end{aligned}
$$

For the sign of $b$ it can be shown that 


$$
\begin{aligned}
& b=\sum_{k, i=1}^{6} v_{k} w_{i} \frac{\partial^{2} f_{k}}{\partial x_{i} \partial \beta^{*}} \\
& =\frac{x_{1}^{*}\left[v_{3} \xi+(1-\xi) v_{4}\right]\left(w_{5} \eta+w_{4}\right)}{x_{1}^{*}+x_{2}{ }^{*}}>0 .
\end{aligned}
$$

Thus, the transformed system (10), or equivalently system (8) undergoes backward bifurcation at $\mathrm{R}_{0}=1$ as required. This result is summarized below.

Theorem 3. The model (8) exhibits backward bifurcation at $\mathrm{R}_{0}=1$ whenever $a>0$ if $\mathrm{B}_{1}>\mathrm{B}_{2}$ and $b>0$.

It should be noted that, in the absence of re-infection (i.e. when $\psi_{\mathrm{e}}=\psi_{\mathrm{c}}=\psi_{\mathrm{r}}=0$ ), the backward bifurcation coefficient, $a$, given in (13) reduces to

$$
\begin{aligned}
a= & \frac{-2 \beta\left(w_{5} \eta+w_{4}\right)}{\left(x_{1}{ }^{*}+x_{2}{ }^{*}\right)^{2}}\left[\left(v_{4} x_{1}^{*}(1-\xi)+v_{3} \xi x_{1}^{*}\right)\left(w_{2}+w_{3}+w_{4}+w_{5}+w_{6}\right)\right. \\
& \left.+w_{1}\left(v_{4} x_{2}{ }^{*}(1-\xi)+v_{3} \xi x_{2}^{*}\right)\right]<0
\end{aligned}
$$

since all the model parameters and the eigenvectors $\mathrm{w}_{\mathrm{i}}(\mathrm{i}=2,3$,

6) and $\mathrm{v}_{\mathrm{i}}(\mathrm{i}=1,2$

6) are nonnegative and $0<\xi<1$. Thus, since the inequality (14) does not hold in this case, the model (8) will not undergo backward bifurcation in the absence of re-infection. This result is summarized below.

Lemma 2. The model (8) does not undergo backward bifurcation at $\mathrm{R}_{0}=1$ in the absence of re infection (i.e. , when $\left.\psi_{\mathrm{e}}=\psi_{\mathrm{c}}=\psi_{\mathrm{r}}=0\right)$.

\section{Global Stability of the DFE of the Model}

The result given in Lemma 2 (discounting the possibility of backward bifurcation when $\psi_{\mathrm{e}}=\psi_{\mathrm{c}}=\psi_{\mathrm{r}}=0$ ) suggests that the DFE, $\varepsilon_{0}$, of the model (8), may be globally asymptotically stable (GAS) when $\mathrm{R}_{0}<1$ and $\psi_{\mathrm{e}}=$ $\psi_{\mathrm{c}}=\psi_{\mathrm{r}}=0$. This is explored below.

Theorem 4. The DFE, $\varepsilon_{0}$, of the model(8) with $\psi_{\mathrm{e}}=\psi_{\mathrm{c}}=\psi_{\mathrm{r}}=0$, is GAS in D if $\mathrm{R}_{0} \leq 1$.

Proof . Consider the Lyapunov function

where,

$$
F=f_{1} L+f_{2} I+f_{3} C
$$

$$
\begin{aligned}
& f_{1}=\omega_{3} k_{3}+\eta \omega_{1} \beta_{2}+\eta \beta_{1} k_{2}, \\
& f_{2}=k_{1} k_{3}-\beta_{1} \mu \omega v+\eta k_{1} \beta_{2}, \\
& f_{3}=\mu \omega v \omega_{1}+\eta k_{1} k_{2}
\end{aligned}
$$

with Lyapunov derivative given by (where a dot represents differentiation with respect to $t$ )

$$
\begin{aligned}
\dot{F}= & f_{1} \dot{L}+f_{2} \dot{I}+f_{3} \dot{C}, \\
= & f_{1}\left[\mu \omega v C+\xi \lambda X-\omega_{1} \varepsilon_{1} L-\beta_{1} \varepsilon_{2} L-\mu_{1} L\right]+f_{2}\left[(1-\xi) \lambda X+\omega_{1} \varepsilon_{1} L-\beta_{2} \varepsilon_{3} I\right. \\
- & \left.\beta_{3} t_{1} I-\mu_{2} I\right]+f_{3}\left[\beta_{1} \varepsilon_{2} L+\beta_{2} \varepsilon_{3} I-\beta_{4} t_{1} C-\mu_{1} C-\mu_{2} C\right], \\
= & \frac{I}{N}\left[N k_{1} k_{2} k_{3}+\beta x_{4} \mu \omega v(1-\xi)\right] \\
& \left(\frac{\beta x_{4}(1-\xi) k_{1} k_{3}+\beta x_{4} \eta \beta_{2}(1-\xi) k_{1}+\beta x_{4} \xi \eta k_{4}+\beta x_{4} \xi \omega_{1} k_{3}+N \mu \omega v k_{4}}{N k_{1} k_{2} k_{3}+\beta_{1}(1-\xi) \beta x_{4} \mu \omega v}-1\right) \\
+ & \frac{1}{N} \eta C\left[N k_{1} k_{2} k_{3}+\beta x_{4} \mu \omega v(1-\xi)\right] \\
& \left(\frac{\beta x_{4}(1-\xi) k_{1} k_{3}+\beta x_{4} \eta \beta_{2}(1-\xi) k_{1}+\beta x_{4} \xi \eta k_{4}+\beta x_{4} \xi \omega_{1} k_{3}+N \mu \omega v k_{4}}{N k_{1} k_{2} k_{3}+\beta_{1}(1-\xi) \beta x_{4} \mu \omega v}-1\right), \\
= & {\left[k_{1} k_{2} k_{3}+\frac{\beta x_{4}}{N} \beta_{1}(1-\xi) \mu \omega v\right]\left[R_{0}-1\right](I+\eta C), }
\end{aligned}
$$


where $\mathrm{x}_{4}$ is susceptible class since $\mathrm{x}_{4} \leq \mathrm{N}$. Thus, $\dot{F} \leq 0$ if $\mathrm{R}_{0} \leq 1$ with $\dot{F}=0$ if and only if $\mathrm{I}=\mathrm{C}=0$ (since $\lambda=\frac{\beta[I+\eta C]}{N}=0$ in this case). It follows, from the LaSalle's Invariance Principle (Lasalle (1976)), that $\mathrm{I} \rightarrow 0$, $\mathrm{C} \rightarrow 0$ as $\mathrm{t} \rightarrow \infty$.

\section{Special Case}

\section{Existence of Endemic Equilibrium Point (EEP):}

In this section, the possible existence and stability of endemic (positive) equilibria of the model (8) (i.e., equilibria where at least one of the infected components of the model is non-zero) will be considered for the special case where exogenous re-infection does not occur (i.e., $\psi_{\mathrm{e}}=\psi_{\mathrm{c}}=\psi_{\mathrm{r}}=0$ ).

Let $\varepsilon_{1}=\left(\mathrm{X}^{* *}, \mathrm{Y}^{* *}, \mathrm{~L}^{* *}, \mathrm{I}^{* *}, \mathrm{C}^{* *}, \mathrm{R}^{* *}\right)$ represents any arbitrary endemic equilibrium of the model (8) with $\psi_{\mathrm{e}}=$ $\psi_{\mathrm{c}}=\psi_{\mathrm{r}}=0$. Solving the equations of the system we have the following endemic equilibrium points (EEP)

$$
\begin{aligned}
\mathrm{X}^{* *}= & \frac{x_{0}}{R_{0}} \\
\mathrm{Y} * *= & \frac{1}{\left(1-\sigma+\mu_{1}\right)^{2}}\left[\mu(1-\omega)+\frac{x_{0} \sigma}{R_{0}}\right] \\
\mathrm{L}^{* *}= & \frac{\mu\left(1-\sigma+\omega \mu_{1}\right)\left[R_{0}-1\right]}{R_{0}\left(1-\sigma+\mu_{1}\right)}\left[\frac{\beta_{2} \mu \omega v(1-\xi)+k_{2} k_{3} \xi+\mu \omega v k_{4}(1-\xi)}{k_{1} k_{2} k_{3}+\mu \omega v k_{1} \beta_{2}(1-\xi)}\right] \\
\mathrm{I}^{* *}= & \frac{\mu\left(1-\sigma+\omega \mu_{1}\right)\left[R_{0}-1\right]}{R_{0}\left(1-\sigma+\mu_{1}\right)}\left[\frac{k_{1} k_{3}(1-\xi)+\xi \omega_{1} k_{3}+\mu \omega v k_{4}(1-\xi)}{k_{1} k_{2} k_{3}+\mu \omega v k_{1} \beta_{2}(1-\xi)+\mu \omega v \beta_{1}(1-\xi)}\right] \\
\mathrm{C}^{* *}= & \frac{\mu\left(1-\sigma+\omega \mu_{1}\right)\left[R_{0}-1\right]}{R_{0}\left(1-\sigma+\mu_{1}\right)}\left[\frac{k_{1} \beta_{2}(1-\xi)+\xi k_{4}+\mu \omega v k_{4}(1-\xi)}{k_{1} k_{2} k_{3}+\mu \omega v k_{1} \beta_{2}(1-\xi)}\right] \\
\mathrm{R}^{* *}= & \frac{\beta_{3}}{\mu_{1}} I^{* *}+\frac{\beta_{4}}{\mu_{1}} C^{* *}
\end{aligned}
$$

So, we have positive endemic equilibrium points only where $\mathrm{R}_{0}>1$. The expression for $\lambda$, at the endemic steady state, denoted by $\lambda^{* *}$, is given by

$$
\lambda^{* *}=\frac{\beta\left(I^{* *}+\eta C^{* *}\right)}{N^{*}} .
$$

Lemma 3. The model (8) with $\psi_{\mathrm{e}}=\psi_{\mathrm{c}}=\psi_{\mathrm{r}}=0$ has a unique endemic equilibrium, given by $\varepsilon_{1}$ whenever $\mathrm{R}_{0}>1$.

\section{Local Stability of EEP: Special Case}

The local stability of the unique EEP, $\varepsilon_{1}$, will now be explored for the special case where the diseaseinduced mortality is negligible (i.e., $\mu_{2}=0$ ), no fast progression to active disease (i.e., $\xi=1$ ) and re-infection does not occur (so that, $\psi_{\mathrm{e}}=\psi_{\mathrm{c}}=\psi_{\mathrm{r}}=0$ ). Setting $\mu_{2}=\psi_{\mathrm{e}}=\psi_{\mathrm{c}}=\psi_{\mathrm{r}}=0$ in the model (8). Using the substitution X $=\mathrm{N}^{*}-\mathrm{Y}-\mathrm{L}-\mathrm{I}-\mathrm{C}-\mathrm{R}$ (and noting that $\mu_{2}=\psi_{\mathrm{e}}=\psi_{\mathrm{c}}=\psi_{\mathrm{r}}=0$ ) and $\xi=1$ in the model (8) gives the following reduced model:

$$
\begin{aligned}
\frac{d Y}{d t} & =\mu(1-\omega)+\sigma\left[\mathrm{N}^{*}-\mathrm{Y}-\mathrm{L}-\mathrm{I}-\mathrm{C}-\mathrm{R}\right]-(1-\sigma) \mathrm{Y}-\mu_{1} \mathrm{Y} \\
\frac{d L}{d t} & =\mu \omega v \mathrm{C}+\frac{\beta(I+\eta C)}{N}\left[\mathrm{~N}^{*}-\mathrm{Y}-\mathrm{L}-\mathrm{I}-\mathrm{C}-\mathrm{R}\right]-\omega_{1} \varepsilon_{1} \mathrm{~L}-\beta_{2} \varepsilon_{2} \mathrm{~L}-\mu_{1} \mathrm{~L} \\
\frac{d I}{d t} & =\omega_{1} \varepsilon_{1} \mathrm{~L}-\beta_{2} \varepsilon_{2} \mathrm{I}-\beta_{3} \mathrm{t}_{1} \mathrm{I} \\
\frac{d C}{d t} & =\beta_{1} \varepsilon_{2} \mathrm{~L}+\beta_{2} \varepsilon_{3} \mathrm{I}-\beta_{4} \mathrm{t}_{1} \mathrm{C}-\mu_{1} \mathrm{C}
\end{aligned}
$$




$$
\frac{d R}{d t}=\beta_{3} \mathrm{t}_{1} \mathrm{I}+\beta_{4} \mathrm{t}_{1} \mathrm{C}-\mu_{1} \mathrm{R},
$$

It is easy to show that the system has a unique EEP, of the form $\varepsilon_{2}=\varepsilon_{1} \mid \mu_{2}=\psi_{e}=\psi_{c}=\psi_{r}=0, \xi=1$, whenever $\mathrm{R}_{01}>1$. We claim the following:

Theorem 5. The unique endemic equilibrium, $\varepsilon_{2}$, of the reduced model is LAS whenever $\mathrm{R}_{01}>1$.

Proof. The proof is based on using the technique in (Hethcote and Thieme (1985)), which employs a Krasnoselskii sub-linearity trick. The approach essentially entails showing that the linearization of the system (17), around the equilibrium $\varepsilon_{2}$, has solutions of the form

$$
\bar{Z}(t)=\bar{Z}_{0} e^{\theta t},
$$

Substituting a solution of the form (18) into the linearized system of (17), around the equilibrium $\varepsilon_{2}$, gives the following linear system

$$
\begin{aligned}
& \theta Z_{1}=(-b-\sigma) Z_{1}-\sigma\left(Z_{2}+Z_{3}+Z_{4}+Z_{5}\right), \\
& \theta Z_{2}=-a_{1} Z_{1}+\left(-a_{1}-k_{1}\right) Z_{2}+\left(a_{2}-a_{1}\right) Z_{3}+\left(\mu \omega v+\eta a_{2}-a_{1}\right) Z_{4}-a_{1} Z_{5}, \\
& \theta Z_{3}=\omega_{1} Z_{2}-k_{2} Z_{3}, \\
& \theta Z_{4}=\beta_{1} Z_{2}+\beta_{2} Z_{3}-k_{3} Z_{4}, \\
& \theta Z_{5}=\beta_{3} Z_{3}+\beta_{4} Z_{4}-\mu_{1} Z_{5} .
\end{aligned}
$$

where, $b=1-\sigma+\mu_{1}, \omega_{1} \varepsilon_{1}=\omega_{1}, \beta_{1} \varepsilon_{2}=\beta_{1}, \beta_{2} \varepsilon_{3}=\beta_{2}, \beta_{3} t_{1}=\beta_{3}, \beta_{4} t_{1}=\beta_{4}, k_{1}=\omega_{1}+\beta_{1}+\mu_{1}, k_{2}=\beta_{2}+\beta_{3}, k_{3}=\beta_{4}+$ $\mu_{1}, a_{1}=\frac{\beta(I+\eta C)}{N}, a_{2}=\frac{\beta X}{N}$

Firstly, all the negative terms in the last three equations of the system(19) are moved to their respective left hand sides. Solving these equations of (19) and substituting the result into the remaining equations and simplifying, gives the equivalent system,

$$
\begin{aligned}
& Z_{1}\left[1+F_{1}(\theta)\right]+Z_{2}\left[1+F_{2}(\theta)\right]=\frac{a_{2}}{k_{1}} Z_{3}+\frac{\mu \omega v+\eta a_{2}}{k_{1}} Z_{4}, \\
& Z_{3}\left[1+F_{3}(\theta)\right]=\frac{\omega_{1}}{k_{2}} Z_{2}, \\
& Z_{4}\left[1+F_{4}(\theta)\right]=\frac{\beta_{1}}{k_{3}} Z_{2}+\frac{\beta_{2}}{k_{3}} Z_{3}, \\
& Z_{5}\left[1+F_{5}(\theta)\right]=\frac{\beta_{3}}{\mu_{1}} Z_{3}+\frac{\beta_{4}}{\mu_{1}} Z_{4},
\end{aligned}
$$

where, $\quad F_{1}(\theta)=\frac{\theta+\sigma}{b}+\frac{a_{1}}{k_{1}}$,

$$
\begin{aligned}
F_{2}(\theta)= & \frac{\theta}{b}+\frac{\theta+a_{1}}{k_{1}}+\left[\frac{\sigma}{b}+\frac{a_{1}}{k_{1}}\right]\left[\frac{\omega_{1}}{\theta+k_{2}}+\frac{\beta_{1}\left(\theta+k_{2}\right)+\omega_{1} \beta_{2}}{\left(\theta+k_{3}\right)\left(\theta+k_{2}\right)}\right]+ \\
& \frac{\beta_{1} \omega_{1}\left(\theta+k_{3}\right)+\beta_{1} \beta_{4}\left(\theta+k_{2}\right)+\omega_{1} \beta_{2} \beta_{4}}{\left(\theta+k_{3}\right)\left(\theta+k_{2}\right)\left(\theta+\mu_{1}\right)}, \\
F_{3}(\theta)= & \frac{\theta}{k_{2}}, F_{4}(\theta)=\frac{\theta}{k_{3}}, F_{5}(\theta)=\frac{\theta}{\mu_{1}},
\end{aligned}
$$


with, $\quad M=\left(\begin{array}{ccccc}0 & 0 & 0 & 0 & 0 \\ 0 & 0 & \frac{a_{2}}{k_{1}} & \frac{\mu \omega v+\eta a_{2}}{k_{1}} & 0 \\ 0 & \frac{\omega_{1}}{k_{2}} & 0 & 0 & 0 \\ 0 & \frac{\beta_{1}}{k_{3}} & \frac{\beta_{2}}{k_{3}} & 0 & 0 \\ 0 & 0 & \frac{\beta_{3}}{\mu_{1}} & \frac{\beta_{4}}{\mu_{1}} & 0\end{array}\right)$,

In the above calculations, the notation $M(\bar{Z})_{i}$ (with $\left.\mathrm{i}=1,2,3,4,5\right)$ denotes the $i$ th co-ordinate of the vector $M(\bar{Z})$. It should further be noted that the matrix $\mathrm{M}$ has nonnegative entries, and the equilibrium $\varepsilon_{2}$ satisfies $\varepsilon_{2}=M \varepsilon_{2}$. Furthermore, since the coordinates of $\varepsilon_{2}$ are all positive, it follows then that if $\bar{Z}$ is a solution of (20), then it is possible to find a minimal positive real number s such that

$$
\|\bar{Z}\| \leq \mathrm{s} \varepsilon_{2}
$$

where, $\|\bar{Z}\|=\left(\left\|Z_{1}\right\|,\left\|Z_{2}\right\|,\left\|Z_{3}\right\|,\left\|Z_{4}\right\|,\left\|Z_{5}\right\|\right)$ with the lexicographic order, and $\|\cdot\|$ is a norm in C. The main goal is to show that $\operatorname{Re}(\theta)<0$. Assume the contrary (i.e., $\operatorname{Re}(\theta) \geq 0$ ). We then need to consider two cases: $\theta=0$ and $\theta \neq 0$. Assume the first case (i.e., $\theta=0$ ). Then, (19) is a homogeneous linear system in the variables $Z_{i}(i=1,2,3,4,5)$. The determinant of the system (19) corresponds to that of the Jacobian of the system (17) evaluated at $\varepsilon_{2}$, which is given by

$$
\Delta=-A_{1}+\mu_{1}(b+\sigma)\left(k_{1} k_{2} k_{3}-\mu \omega v k_{4}\right)\left[R_{01} \frac{X^{*}}{N^{*}}-1\right]
$$

where, $A_{1}=b \mu_{1} a_{1} k_{4}+b a_{1} \omega_{1} \beta_{3} k_{3}+b a_{1} \mu_{1} k_{2} k_{3}+b a_{1} \mu_{1} \omega_{1} k_{3}+b a_{1} \beta_{4} k_{4}$ and $k_{1} k_{2} k_{3}-\mu \omega v k_{4}>0$ ( we proved in section 3 ).

It can be shown that $\frac{X^{*}}{N^{*}}=\frac{1}{R_{01}}$. Thus, $\Delta<0$. Consequently, the system (19) can only have the trivial solution $\bar{Z}=\overline{0}$ (which corresponds to the DFE, $\varepsilon_{0}$ ). Consider, next, the case $\theta \neq 0$. In this case, $\operatorname{Re}\left(\mathrm{F}_{\mathrm{i}}(\theta)\right) \geq 0(\mathrm{i}=1,2$, $3,4,5)$ since, by assumption, $\operatorname{Re}(\theta) \geq 0$. It is easy to see that this implies $\left|1+\mathrm{F}_{\mathrm{i}}(\theta)\right|>1$ for all i. Define $\mathrm{F}(\theta)=$ $\min \left|1+\mathrm{F}_{\mathrm{i}}(\theta)\right|>1$; (for $\left.\mathrm{i}=1,2,3,4,5\right)$. Then, $\mathrm{F}(\theta)>1$. Hence, $\frac{s}{F(\theta)}<s$. The minimality of s implies that $\|\bar{Z}\|>\frac{s}{F(\theta)} \varepsilon_{2}$. On the other hand, taking norms of both sides of the third equation of (19), and using the fact that the matrix $M$ is non-negative, gives

$$
F(\theta)\left\|Z_{3}\right\| \leq M(\|Z\|)_{3} \leq s\left(M\left\|\varepsilon_{2}\right\|\right)_{3} \leq s I^{* *} .
$$

Then, it follows from the above inequality that $\left\|Z_{3}\right\| \leq \frac{s}{F(\theta)} I^{* *}$, which contradicts $\operatorname{Re}\left(\mathrm{F}_{\mathrm{i}}(\theta)\right) \geq 0$. Hence, $\operatorname{Re}(\theta)<0$, so that the endemic equilibrium, $\varepsilon_{2}$, is LAS if $\mathrm{R}_{01}>1$.

The epidemiological implication of Theorem 5 is that the disease will persist in the community if the reproduction threshold $\left(\mathrm{R}_{01}\right)$ exceeds unity.

\section{Global Stability of EEP: Special Case}

The global asymptotic stability of EEP, $\varepsilon_{2}$, of the reduced model (17) is considered for the case when after taking vaccination some people get complete immunity (i.e. the unsuccessful vaccination rate $(1-\sigma)=0$ ). 
Setting $(1-\sigma)=0$ into the reduced model (17), and using the substitution $\beta_{0}=\frac{\beta}{N^{*}}$, it can be shown that the associated reproduction number of the reduced model (17) with $\xi=1$ and $(1-\sigma)=0$, denoted by $\mathrm{R}_{02}$, is given by

$$
R_{02}=\frac{\eta \beta_{0} x_{4} k_{4}+\mu \omega v k_{4}+\beta_{0} x_{4} \omega_{1} k_{3}}{k_{1} k_{2} k_{3}}
$$

where $\mathrm{x}_{4}, \mathrm{k}_{1}, \mathrm{k}_{2}, \mathrm{k}_{3}$ are defined in section 3. Furthermore, using the approach in Section 4, it can be shown that the reduced system (17), with $(1-\sigma)=0$, has a unique EEP, of the form

$$
\varepsilon_{3}=\left.\varepsilon_{2}\right|_{(1-\sigma)=0}=\mathrm{X}^{* *}, \mathrm{Y}^{* *}, \mathrm{~L}^{* *}, \mathrm{I}^{* *}, \mathrm{C}^{* *}, \mathrm{R}^{* *},
$$

where, $\mathrm{X}^{* *}>0, \mathrm{Y}^{* *}>0, \mathrm{~L}^{* *}>0, \mathrm{I}^{* *}>0, \mathrm{C}^{* *}>0, \mathrm{R}^{* *}>0$ whenever $\mathrm{R}_{02}>1$.

Let, $\mathrm{D}_{0}=\{(\mathrm{X}, \mathrm{Y}, \mathrm{L}, \mathrm{I}, \mathrm{C}, \mathrm{R}) \in \mathrm{D}: \mathrm{L}=\mathrm{I}=\mathrm{C}=\mathrm{R}=0\}$.

Theorem 6. The unique EEP, $\varepsilon_{3}$, of the reduced model (17) with $(1-\sigma)=0$, is GAS in $D \backslash D_{0}$ whenever $R_{02}>1$. Proof . Let $(1-\sigma)=0$ and $R_{02}>1$, so that the EEP, $\varepsilon_{3}$, exists. Consider the following non-linear Lyapunov function:

$$
\begin{aligned}
L= & \left(X-X^{* *}-X^{* *} \ln \frac{X}{X^{* *}}\right)+\left(L-L^{* *}-L^{* *} \ln \frac{L}{L^{* *}}\right) \\
& +\frac{\beta_{0} X^{* *}\left[\left(\mu_{1}+\beta_{4}\right)+\eta \beta_{2}\right]}{\left(\mu_{1}+\beta_{4}\right)\left(\beta_{2}+\beta_{3}\right)}\left(I-I^{* *}-I^{* *} \ln \frac{I}{I^{* *}}\right)+\frac{\beta_{0} \eta X^{* *}}{\mu_{1}+\beta_{4}}\left(C-C^{* *}-C^{* *} \ln \frac{C}{C^{* *}}\right)
\end{aligned}
$$

with Lyapunov derivative given by,

$$
\begin{aligned}
& \dot{L}\left(1-\frac{X^{* *}}{X}\right) \dot{X}+\left(1-\frac{L^{* *}}{L}\right) \dot{L}+\frac{\beta_{0} X^{* *}\left[\left(\mu_{1}+\beta_{4}\right)+\eta \beta_{2}\right]}{\left(\mu_{1}+\beta_{4}\right)\left(\beta_{2}+\beta_{3}\right)}\left(1-\frac{I^{* *}}{I}\right) \dot{I}+\frac{\beta_{0} \eta X^{* *}}{\mu_{1}+\beta_{4}} \\
&\left(1-\frac{C^{* *}}{C}\right) \dot{C}, \\
&=\left(1-\frac{X^{* *}}{X}\right)\left[\mu \omega v C^{* *}+\beta_{0} I^{* *} X^{* *}+\beta_{0} \eta C^{* *} X^{* *}+\sigma X^{* *}+\mu_{1} X^{* *}-\mu \omega v C-\beta_{0} I X-\beta_{0} \eta C X\right. \\
&-\left.\sigma X+\mu_{1} X\right]+\left(1-\frac{L^{* *}}{L}\right)\left[\mu \omega v C+\beta_{0} I X+\beta_{0} \eta C X-\omega_{1} L-\beta_{1} L-\mu_{1} L\right]+ \\
& \frac{\beta_{0} X^{* *}\left[\left(\mu_{1}+\beta_{4}\right)+\eta \beta_{2}\right]}{\left(\mu_{1}+\beta_{4}\right)\left(\beta_{2}+\beta_{3}\right)}\left(1-\frac{I^{* *}}{I}\right)\left[\omega_{1} L-\beta_{2} I-\beta_{3} I\right]+\frac{\beta_{0} \eta X^{* *}}{\mu_{1}+\beta_{4}}\left(1-\frac{C^{* *}}{C}\right)\left[\beta_{1} L+\beta_{2} I-\beta_{4} C-\mu_{1} C\right],
\end{aligned}
$$




$$
\begin{aligned}
= & \mu \omega \nu C^{* *}+\beta_{0} I^{* *} X^{* *}+\beta_{0} \eta C^{* *} X^{* *}+\sigma X^{* *}+\mu_{1} X^{* *}-\sigma X-\mu_{1} X-\mu \omega \nu C^{* *} \frac{X^{* *}}{X} \\
& -\beta_{0} I^{* *} X^{* *} \frac{X^{* *}}{X}-\beta_{0} \eta C^{* *} X^{* *} \frac{X^{* *}}{X}-\sigma X^{* *} \frac{X^{* *}}{X}-\mu_{1} X^{* *} \frac{X^{* *}}{X}+\mu \omega \nu C \frac{X^{* *}}{X}+\beta_{0} X^{* *}
\end{aligned}
$$

$+\beta_{0} \eta C X^{* *}+\sigma X^{* *}+\mu_{1} X^{* *}-\left(\omega_{1}+\beta_{1}+\mu_{1}\right) L-\mu \omega \nu C \frac{L^{* *}}{L}-\beta_{0} \eta C X \frac{L^{* *}}{L}+\left(\omega_{1}+\beta_{1}+\mu_{1}\right) L^{* *}$

$+\frac{\beta_{0} \omega_{1}}{\beta_{2}+\beta_{3}}\left[L X^{* *}-L X^{* *} \frac{I^{* *}}{I}\right]-\beta_{0} L X^{* *}+\beta_{0} I^{* *} X^{* *}+\frac{\eta \omega_{1} \beta_{0} \beta_{2}}{\left(\mu_{1}+\beta_{4}\right)\left(\beta_{2}+\beta_{3}\right)}\left[L X^{* *}-L X^{* *} \frac{I^{* *}}{I}\right]$

$+\frac{\eta \beta_{0} \beta_{2}}{\mu_{1}+\beta_{4}}\left[I^{* *} X^{* *}-X^{* *} I\right]+\frac{\eta \beta_{0} \beta_{1}}{\mu_{1}+\beta_{4}}\left[L X^{* *}-L X^{* *} \frac{C^{* *}}{C}\right]+\frac{\eta \beta_{0} \beta_{2}}{\mu_{1}+\beta_{4}}\left[I X^{* *}-I X^{* *} \frac{C^{* *}}{C}\right]$

$-\beta_{0} \eta C X^{* *}+\beta_{0} \eta C^{* *} X^{* *}$,

$=\mu \omega \nu C^{* *}+\beta_{0} I^{* *} X^{* *}+\beta_{0} \eta C^{* *} X^{* *}+\sigma X^{* *}+\mu_{1} X^{* *}-\sigma X-\mu_{1} X-\mu \omega \nu C^{* *} \frac{X^{* *}}{X}$

$-\beta_{0} I^{* *} X^{* *} \frac{X^{* *}}{X}-\beta_{0} \eta C^{* *} X^{* *} \frac{X^{* *}}{X}-\sigma X^{* *} \frac{X^{* *}}{X}-\mu_{1} X^{* *} \frac{X^{* *}}{X}-\beta_{0} I^{* *} X^{* *} \frac{X^{* *}}{X} \frac{C}{C^{* *}}$

$-\beta_{0} \eta C X^{* *} \frac{X^{* *}}{X}+\mu \omega \nu C^{* *} \frac{C}{C^{* *}} \frac{X^{* *}}{X}+\beta_{0} I^{* *} X^{* *} \frac{X^{* *}}{X} \frac{C}{C^{* *}}+\beta_{0} \eta C^{* *} X^{* *} \frac{X^{* *}}{X} \frac{C}{C^{* *}}+\sigma X^{* *}$

$+\mu_{1} X^{* *}-\mu \omega \nu C^{* *} \frac{L}{L^{* *}}-\beta_{0} I^{* *} X^{* *} \frac{L}{L^{* *}}-\beta_{0} \eta C^{* *} X^{* *} \frac{L}{L^{* *}}-\mu \omega \nu C^{* *} \frac{L^{* *}}{L}-\beta_{0} I X \frac{L^{* *}}{L}$

$-\beta_{0} \eta C X \frac{L^{* *}}{L}+\mu \omega v C^{* *}+\beta_{0} I^{* *} X^{* *}+\beta_{0} \eta C^{* *} X^{* *}+\frac{\beta_{0} I^{* *}}{L^{* *}}\left[L X^{* *}-L X^{* *} \frac{I^{* *}}{I}\right]+\beta_{0} I^{* *} X^{* *}$

$\frac{\beta_{0} \eta C^{* *}}{L^{* *}}\left[L X^{* *}-L X^{* *} \frac{I^{* *}}{I}\right]+\frac{\beta_{0} \eta C^{* *}}{I^{* *}}\left[I^{* *} X^{* *}-X^{* *} I\right]+\frac{\beta_{0} \eta C^{* *}}{L^{* *}}\left[L X^{* *}-L X^{* *} \frac{C^{* *}}{C}\right]$

$+\frac{\beta_{0} \eta C^{* *}}{I^{* *}}\left[I X^{* *}-I X^{* *} \frac{C^{* *}}{C}\right]+\beta_{0} \eta C^{* *} X^{* *}$,

$=\sigma X^{* *}\left[2-\frac{X}{X^{* *}}-\frac{X^{* *}}{X}\right]+\mu_{1} X^{* *}\left[2-\frac{X}{X^{* *}}-\frac{X^{* *}}{X}\right]+$

$\beta_{0} I^{* *} X^{* *}\left[3-\frac{X^{* *}}{X}-\frac{L}{L^{* *}} \frac{I^{* *}}{I}-\frac{X}{X^{* *}} \frac{L^{* *}}{L} \frac{I}{I^{* *}}\right]+$

$\beta_{0} \eta C^{* *} X^{* *}\left[4-\frac{X^{* *}}{X}-\frac{L}{L^{* *}} \frac{C^{* *}}{C}-\frac{I}{I^{* *}} \frac{C^{* *}}{C}-\frac{X}{X^{* *}} \frac{L^{* *}}{L} \frac{C}{C^{* *}}+\frac{L}{L^{* *}}-\frac{L}{L^{* *}} \frac{I^{* *}}{I}\right]+$

$\mu \omega v C^{* *}\left[2-\frac{X^{* *}}{X}+\frac{C}{C^{* *}} \frac{X^{* *}}{X}-\frac{L}{L^{* *}}-\frac{L^{* *}}{L}\right]$, 
$=\sigma X^{* *}\left[2-\frac{X}{X^{* *}}-\frac{X^{* *}}{X}\right]+\mu_{1} X^{* *}\left[2-\frac{X}{X^{* *}}-\frac{X^{* *}}{X}\right]+\mu \omega \nu C^{* *}\left[2-\frac{L}{L^{* *}}-\frac{L^{* *}}{L}\right]$

$+\beta_{0} I^{* *} X^{* *}\left[3-\frac{X^{* *}}{X}-\frac{L}{L^{* *}} \frac{I^{* *}}{I}-\frac{X}{X^{* *}} \frac{L^{* *}}{L} \frac{I}{I^{* *}}\right]+$

$\beta_{0} \eta C^{* *} X^{* *}\left[4-\frac{X^{* *}}{X}-\frac{I}{I^{* *}} \frac{C^{* *}}{C}-\frac{X}{X^{* *}} \frac{L^{* *}}{L} \frac{C}{C^{* *}}-\frac{L}{L^{* *}} \frac{I^{* *}}{I}\right]$

Since the arithmetic mean exceeds the geometric mean, it follows then that

$$
\begin{aligned}
& 2-\frac{X}{X^{* *}}-\frac{X^{* *}}{X} \leq 0, \\
& 2-\frac{L}{L^{* *}}-\frac{L^{* *}}{L} \leq 0, \\
& 3-\frac{X^{* *}}{X}-\frac{L}{L^{* *}} \frac{I^{* *}}{I}-\frac{X}{X^{* *}} \frac{L^{* *}}{L} \frac{I}{I^{* *}} \leq 0, \\
& 4-\frac{X^{* *}}{X}-\frac{L}{L^{* *}} \frac{I^{* *}}{I}-\frac{I}{I^{* *}} \frac{C^{* *}}{C}-\frac{X}{X^{* *}} \frac{L^{* *}}{L} \frac{C}{C^{* *}} \leq 0,
\end{aligned}
$$

Again, from the above expression we have,

$$
\begin{aligned}
& \beta_{0} \eta C^{* *} X^{* *}\left[\frac{L}{L^{* *}}-\frac{L}{L^{* *}} \frac{C^{* *}}{C}\right]+\mu \omega v C^{* *}\left[-\frac{X^{* *}}{X}+\frac{C}{C^{* *}} \frac{X^{* *}}{X}\right] \\
& =\beta_{0} \eta C^{* *} X^{* *} \frac{L}{L^{* *}}\left(1-\frac{C^{* *}}{C}\right)-\mu \omega \nu C^{* *} \frac{X^{* *}}{X}\left(1-\frac{C}{C^{* *}}\right)
\end{aligned}
$$

Here, if we consider $\frac{C}{C^{* *}} \leq 1$, then the sign of $\mu \omega v C^{* *} \frac{X^{* *}}{X}\left(1-\frac{C}{C^{* *}}\right)$ is positive and the sign of $\beta_{0} \eta C^{* *} X^{* *} \frac{L}{L^{* *}}\left(1-\frac{C^{* *}}{C}\right)$ is negative. So that $\dot{L} \leq 0$ for $\mathrm{R}_{02}>1$. Hence, $L$ is a Lyapunov function of the system (17), with $(1-\sigma)=0$, on $\mathrm{D} \backslash \mathrm{D}_{0}$. Thus, by the Lyapunov function $L$ and the LaSalle's Invariance Principle, every solution to the equations in the reduced model (17) approaches $\varepsilon_{3}$ as $\mathrm{t} \rightarrow \infty$ for $\mathrm{R}_{02}>1$.

\section{Numerical Simulations and Discussions}

In this section, we have drawn some graphical presentations using data from Table 2. Here we have collected some data from Bangabandhu Shekh Mujibur Rahman Medical University and we assumed some data for our convenience. In figure 1 , prevalence is slowly decreases with vaccination when $\mathrm{R}_{0}=0.8595$ and also decreases without vaccination when $\mathrm{R}_{0}=0.8611$. In figure 2 , prevalence is very high when $\mathrm{R}_{0}=1.1204$ in absence of vaccination and prevalence is gradually increasing when $R_{0}=1.1101$ in presence of vaccination. In figure 3 , total infection decreases when $\mathrm{R}_{0}=0.8611$ in absence of vaccination and also decreases and reduces to zero when $\mathrm{R}_{0}=0.8595$ in presence of vaccination. In figure 4 , total infection is highly increases without vaccination when $R_{0}=1.1204$ and with vaccination, at the beginning disease increasing and smoothly increases when $\mathrm{R}_{0}=1.1101$. From which it is evident that the vaccine has a positive impact, since it reduces the number of infection in comparison to the case when the vaccination is not used. 


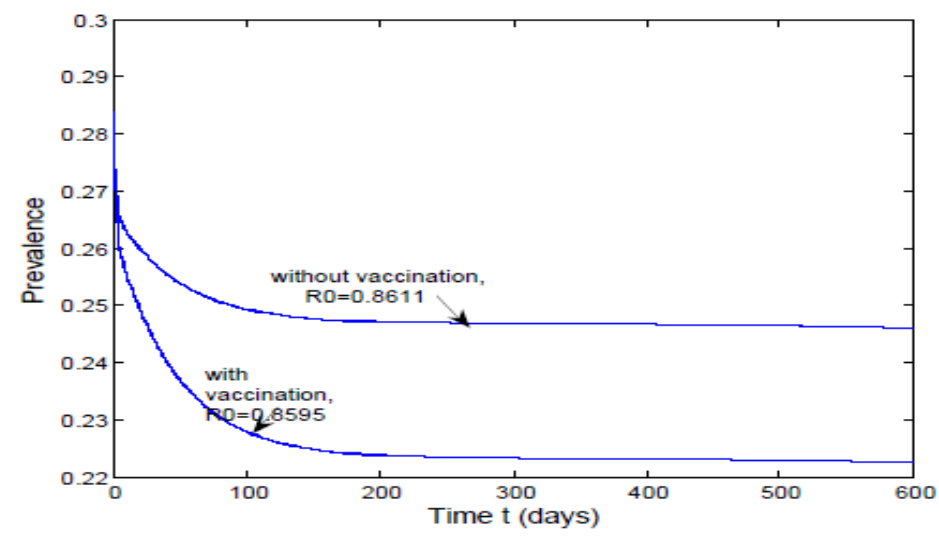

Figure 1: Prevalence in presence and absence of vaccination when $\mathrm{R}_{0}<1$.

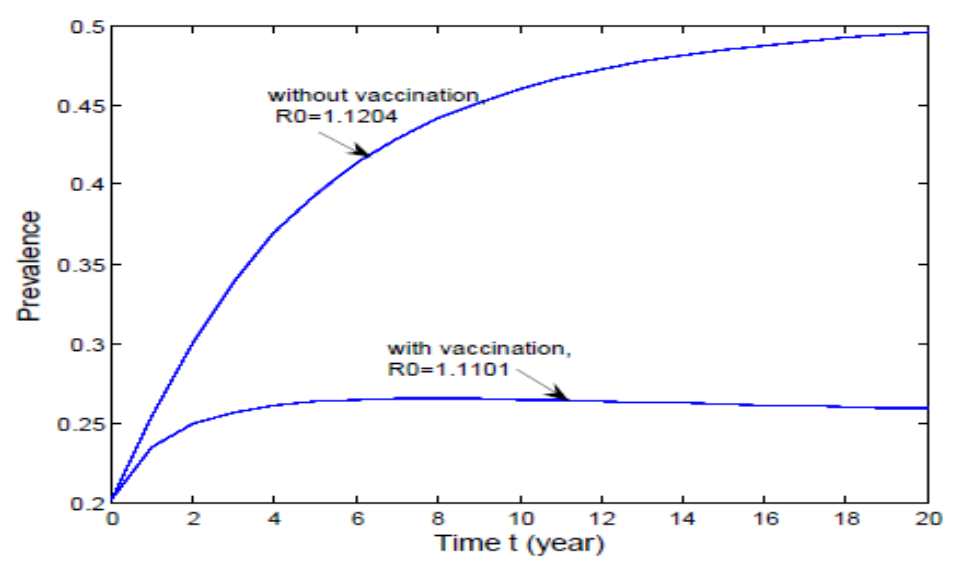

Figure 2: Prevalence in presence and absence of vaccination when $\mathrm{R}_{0}>1$.

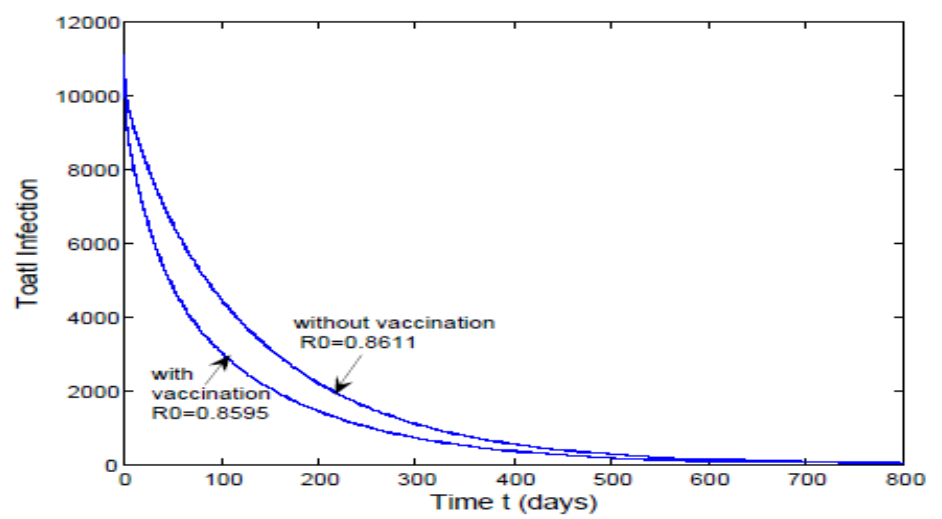

Figure 3: Total infection in presence and absence of vaccination when $\mathrm{R}_{0}<1$. 


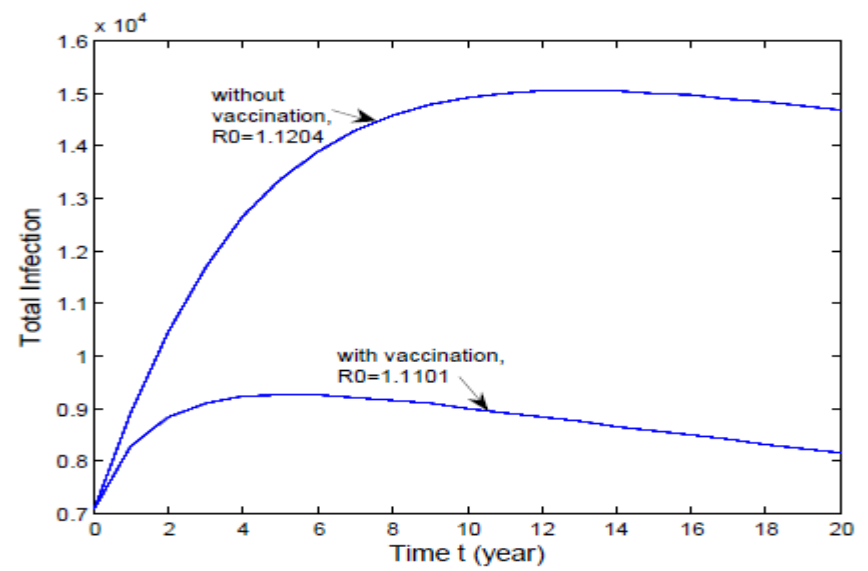

Figure 4: Total infection in presence and absence of vaccination when $\mathrm{R}_{0}>1$.

Here some more graphs on prevalence and infection on different $\mathrm{R}_{0}$ where parameter values varies

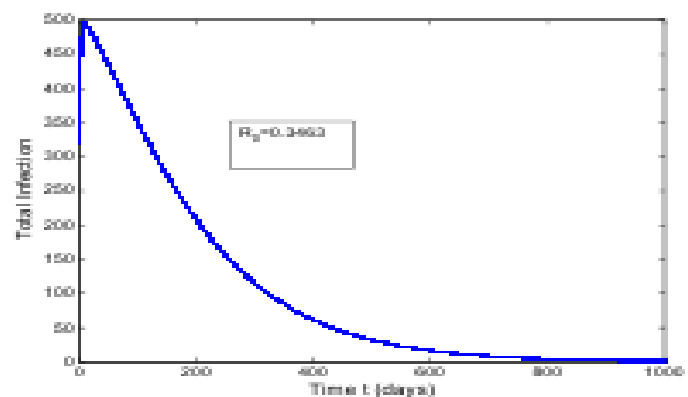

Figure 1: Total infection when $R_{0}=0.3463$

Figure 1: Total infection when $\mathrm{R}_{0}=0.3463$

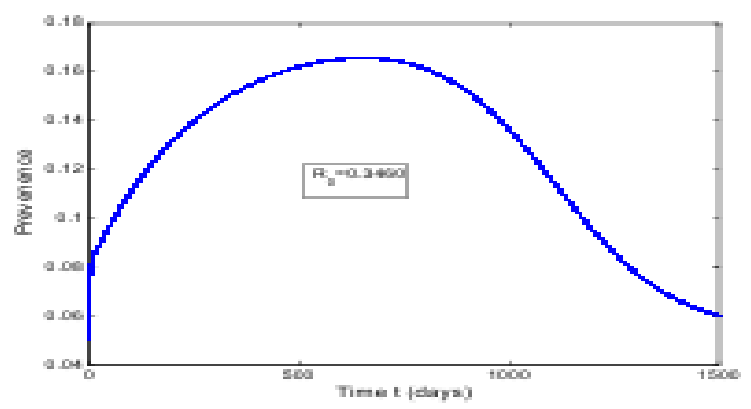

Figure 2: Prevelence when $R_{0}=0.3463$

Figure 2: Total infection when $\mathrm{R}_{0}=0.3463$.

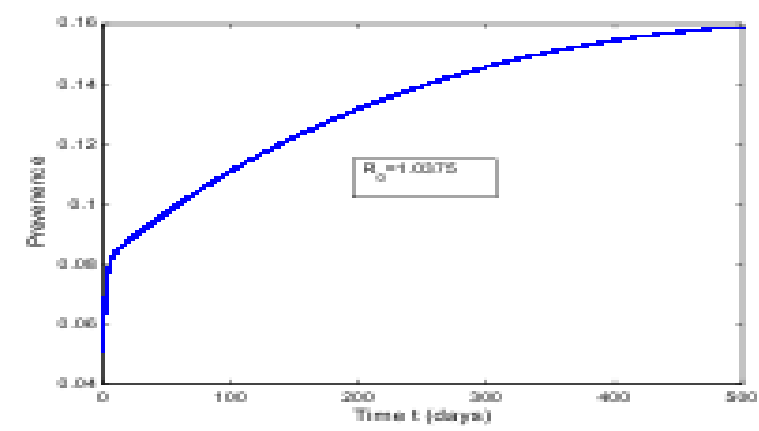

Figure 3: Total infection when $R_{0}=1.0342$

Figure 3: Total infection when $\mathrm{R}_{0}=1.0342$. 


\section{Result}

We rigorously analyzed (mathematically and numerically) the dynamics of HBV in our model. Some mathematical and epidemiological findings of our study are given below:

1. The model has a disease free equilibrium (DFE) which is asymptotically stable if $R_{0}<1$ and unstable if $R_{0}>1$. We found that, the model has a unique endemic equilibrium point and discuss the local stability at EEP using Sub-linearity trick when $R_{0}>1$ and globally asymptotically stable for a special case when $(1-\sigma)=0$

2. The infection decreases when $R_{0}=0.3463<1$.

3. At the same state (i.e. when $R_{0}=0.3463<1$ ) prevalence increases and after a certain time it smoothly decreases.

4. And lastly, the prevalence is very high when $R_{0}=1.0342>1$.

\section{Conclusions}

A deterministic model for the transmission dynamics of HBV in a population is designed and rigorously analyzed. Some of the main findings of the study include the following:

(i) The model exhibits a phenomenon of backward bifurcation, where a stable DFE co-exists with a suitable EEP when the associated reproduction number is less than unity. In the absence of re-infection the DFE is globally asymptotically stable whenever its associated reproduction number is less than unity. The model has an EEE which is globally asymptotically stable for a special case.

(ii) The model does not undergo backward bifurcation when re-infection terms are neglected.

\section{Acknowledgement}

This work has been funded by Bose Centre for Advanced Study and Research in Natural Sciences under the University of Dhaka. We would like to express our special appreciation and thanks for their support.

\section{References}

[1]. A.S. Perelson, A. U. Neumann, M. Markowitz (1996). HIV-1 dynamics in vivo: Virion clearance rate, infested cell lifespan and viral generation time, Science Vol 271, pp: 1582-1586.

[2]. Ahmad N, Alam S, Mustafa G, Adnan AB, Baiq RH, Khan M (2008). e-antigen-negative chronic hepatitis B in Bangladesh. Hepatobiliary Pancreat Dis Int. Vol 7, No 4, pp:379-82.

[3]. Castillo-Chavez, C. and B. Song (2004).Dynamical models of tuberculosis and their applications. Math. Biosci. Eng. Vol 1, No. 2, pp: $361-404$

[4]. Chowdhury B.O., Ahamad M. S. U. Farooque A. H. M. O., Rahman A. J. E. N (2009). Study on association of Hepatocellular Carcinoma with Hepatitis B Virus Infection: Bangladesh Perspective. Journal of Chittagong Medical College Teaches Association. Vol 20, No.1, pp: 31-33.

[5]. Gibney L, Saquib N (2001). Human immunodeficiency virus, hepatitis B, C and D in Bangladesh's trucking industry: prevalence and risk factors. International Journal of Epidemiology; Vol 30, pp: 878-884.

[6]. H.W. Hethcote and H. R. Thieme (1985). Stability of the endemic equilibrium in epidemic models with subpopulations , Math. Biosci. Vol 75, pp: 205-227.

[7]. Hsu Hy, Chang MH, Lee Cy, et al (1992). Spontaneous loss of HBsAg in children with chronic hepatitis B virus infection. Hepatology, Vol 15, pp: 382.

[8]. Islam MN, Islam KM, Islam N. (1984). Hepatitis-B virus infection in Dhaka, Bangladesh. Bangladesh Med Res Counc Bull; Vol 10, pp: 1-6.

[9]. J Dushoff, W. Huang and C. Castillo-Chavez (1998). Backward bifurcations and catastrophe in simple models of fatal diseases, J. Math. Biol. Vol 36, pp: 227-248.

[10]. J. Carr (1981). Application of Centre Manifold Theory, Springer-Verlag, New York.

[11]. J.P. Lasalle (1976), The Stability of Dynamical Systems, Regional Conference Ser. Appl. Math. SIAM, Philadelphia.

[12]. Jianhua Pang, Jing-an Cui, Xueyong Zhon (2010). Dynamical behavior of a hepatitis B virus transmission model with vaccination; Journal of theoretical Biology .Vol 265, pp: 572-578.

[13]. Khan M, Ahmad N (1996). Seroepidemiology of HBV and HCV in Bangladesh. International Hepatology Communications; Vol 5, pp: 27-29.

[14]. L. Esteva A.B. Gumel and C. Vargas de leon (2009). Qualitative study of transmission dynamics of drug-resistant malaria, Math. Comput. Modeling.Vol 50(3, 4), pp: 611-630.

[15]. Lan Zou, Weinian Zhang, Shigui Ruan (2010). Modeling the transmission dynamics and control of hepatitis B virus in China; Journal of Theoretical Biology, Vol 262, pp: 330-338.

[16]. Lok AS, Health cote EJ, Hoofnagle JH (2001). Management of Hepatitis B and summary of a workshop. Gastroenterology ;No. 120, pp: 1828-53.

[17]. M A Ahad, M A Alim (2006). Current Challenges in Hepatitis B, TAJ, Vol 19 ,No. 1.

[18]. Mandell, G.L., Douglas, R.G., Bennett, J.E. (1979). Principles and Practice of infectious Diseases. A Wiley Medical Publication John Wiley and Sons, New York.

[19]. Most. Nazma Parvin, Riaz Uddin and Sadia Afreen Chowdhury (2011). Hepatitis in Bangladesh: Pattern and treatment options. Journal of Applied Pharmaceutical Science, Vol 01, No.06, pp: 118-121. 
[20]. P. D. LeenhEEr, H. L. Smith (2003). Virus dynamics: A global analysis, SIAM J. appl. Math. Vol 63, pp: 131-1327.

[21]. P. van den Driessche and J. Watmough (2002). Reproduction numbers and sub-thresold endemic equilibria for compartmental models of disease transmission, Math. Biosci. Vol 180, pp: 29-48.

[22]. Shepard, C.W., Simard, E.P., et al.,(2006). Hepatitis B virus infection: epidemiology and vaccination. Epidemiologic Reviews 28 , pp: $112-125$.

[23]. Shonjun Zhaoa, b, Zhiyi Xna and Ying Lub (2010). A mathematical model of hepatitis B virus transmission and its application for vaccination strategy in China ; Department of Radiology; University of California, San Francisco, CA 94143-1349; USA.

[24]. Tibbs C.J. and Smith H.M. (2001) Clinicians guide to viral hepatitis. 1st Edition.

[25]. World Health Organization (1998). Communicable/ Infections Disease: Hepatitis B. WHO Fact Sheet.204. 